D a mour, üb. d. eisenhalt. Kupferhydrosilicat. 351

nach dem neueren Standpunct der Wissenschaft veranstalteten Analysen.

Soll zwischen dem Friedrichshaller Soolenwasser und einem andern Mineralwasser ein Vergleich gewagt werden, so dürfte dazu das PülInaer Bitterwasser am geeignetsten sein, doch ist der Salzgehalt des FriedrichshallerWassers quantitativ geringer; ein besserer Vergleich kommt heraus, wenn man sich Püllnaer Bitterwasser und Seewasser zusammengemischt denkt, aber auch hier fehlt es an der verhältnissmåssigen Quantität der gegebenen Hauptsalze.

Gesetzt aber, das Friedrichshaller Wasser soltte als Ersatzmittel für Seewasser als Bad gebraucht werden und man wünschte dasselbe von der Stärke (dem specifischen Gewichte) des Seewassers, so kann diesem Verlangen in so fern Genügo geleistet werden, als man gegebenenfalls nur eine zu bestimmende Quantităt des auf der Saline in Massen vorräthigen Kochsalzes in dem Badewasser aufzulösen hätte. Auch kann za dem Behuf gradirte Soole genommen werden. Wenn man übrigens in Zweifel stellt, ob das Friedrichshaller Wasser auch innerlich genommen werden könne, weil es wenig Kohlensăure in seiner Mischung habe, so habe ich einzuwenden, dass fast alle bekannten Bitterwässer, wie Saidschütz, Püllna, auch nicht mehr oder sehr wenig mebr Kohlensäure haben und doch fast blos innerlich angewendet werden, häufig mit dem glänzendsten Erfolge.

\title{
XLVII.
}

Ueber das eisenhallige Kupferhydrosilicat aus Sibirien. Von

\section{A. D $\triangle \mathbf{M} \quad 0$ U $\mathbf{R}$.}

(Annales des Mines, troisième série, tome XII. Ste Lieferung, 1837. S. 241.)

Dieses Mineral besteht aus amorphen Massen von dunkelbrauner Farbe, und es laufen nach allen Richtungen kleine Adern von grünem kohlensaurem Kupfer and von dunkelgrünem Kupferhydrosilicat durch dasselbe. Sein Pulver besitzt eine gelbe, ins Braune fallende Farbe; es ist zerbrechlich and man 


\section{Dam o ur, üb. d. eisenhalt. Kupferhydrosilicat.}

kann es mit einer Stahlspitze leicht ritzen. Bis zum Rothglüben in einer verschlossenen Röhre erhitzt, entwickelt es viel Wasser, wobei es ungeführ 20 Procent seines Gewichts verliert. Nach dieser Operation lässt es sich -vom Magnet anziehen. Auf Kohle der Flamme des Löthrohres ausgesetzt, bleibt es unschmelzbar, löst sich zum Theil in dem Phosphorsalze aup, welches es blangrün färbt, und hinterlässt cin Kieselskelett. Concentrirte Salzsäure greift es leicht an. Die Kieselerde bleibt allein unlöslich und setyt sich in leichten Flocken ab.

Ich stellte mit diesem Mineral zwei Analysen an, die erste mit einem, die zweite mit zwei Grammen. Sie gaben mir folgende Resultate :

1ste Analyse.

2te Analyse.

Saverstoff.

\begin{tabular}{llllll} 
Kieselerde & $\mathbf{0 , 1 7 7}$ & $\mathbf{0 , 0 9 1 9 5 1}$ & Kieselerde & $\mathbf{0 , 3 6 4}$ \\
Kupferoxyd & $\mathbf{0 , 1 2 0}$ & $\mathbf{0 , 0 2 4 2 0 8}$ & Kupferoxyd & $\mathbf{0 , 2 4 5}$ \\
Eisenoxyd & $\mathbf{0 , 4 9 2}$ & $\mathbf{0 , 1 5 0 8 5 4}$ & Eisenoxyd & $\mathbf{1 , 0 5 0}$ \\
Wasser & $\mathbf{0 , 2 0 6}$ & $\mathbf{0 , 1 8 3 1 1 1}$ & Wasser & $\mathbf{0 , 4 1 0}$ \\
\cline { 2 - 2 } & $\mathbf{0 , 9 9 5 .}$ & & & $\mathbf{2 , 0 6 9 .}$
\end{tabular}

Das Wasser wurde besonders bei einer Quantitit von 1 Gramm bestimmt. Ioh traf mit der in Relie stehenden Substanz eine genaue Auswahl, indem ich mich einer Loupe bediente, am die sichtbare Beimengung von kieselsaurem oder kohlensaurem Kupfer zu entfernen.

Das Mineral wurde in kleinen Stücken unter einer Glocke getrocknet, welche über einer mit concentrirter Schwefelsäure gefüllten Schale stand. Nachher wurde es in einem bedeckten Platintiegel gewogen, darauf mit Vorsicht der Rothglühhitze ausgesetzt. Bei dieser Operation verlor es 0,206 Gr.

Ehe die Mengen der anderen Bestandtheile des Minerals bestimmt wurden, suchte ich mich zu überzeugen, ob das darin enthaltene Eisen sich als Eisenoxydul oder als Eisenoxyd darin befände. Ich löste daher mittelst verdünnter Salzsäure einen Theil der Substanz auf, der auf den Boden eines genau angchliessenden und mit Kohlensüure zuvor angefüllten Ballons gebracht wurde. Die Lösung wurde mit Wasser verdünnt, das von Luft befreit war, und das Zusetzen einiger Tropfen Kaliumeisencyanid erzeugte darin einen ziemlich reichlichen Niederschlag von gelber, ins Grüne schielenden Farbe. Dieser 
Damour, üb. d. eisenhalt. Kupferhydrosilicat. $\mathbf{3 5 3}$

kam fast ganz von der Anwesenheit des Kupferoxydes her, seine grünliche Farbe aber liess vermuthen, dass sich Berlinerblau gebildet habe, das von der Anwesenheit einer kleinen Menge Eisenoxydul herrühre. Ein anderer Theil der Substanz wurde unter den nämlichen Vorsichtsmaassregeln in Salzsäure aufgelöst, darauf mit Chlorgoldnatrium gemischt. Ich konnte darin keine merkliche Spur von reducirtem Golde entdecken. Diese Resultate leiteten mich zu dem Schlusse, dass das Eisen sich in diesem Minerale als Eisenoxyd vorfinde, das wahrscheinlich einige Spuren von Eisenoxydul enthalte.

Ein Gramm trockenes Pulver des Minerals warde darauf in concentrirter Salzsäure aufgelöst, die Auflösung bis zur Trockne abgedampft und wieder mit schwacher Salzsäure behandelt und filtrirt. Es blieben 0,177 Gr. Kieselerde zurück. Die filtrirte Flüssigkeit wurde der Wirkung eines Stromes Schwefelwasserstoff unterworfen. Der erhaltene aus Schwefelkupfer bestehende Niederschlag wurde auf einem Filter gesammelt, mit Wasser gewaschen, das mit Schwerelwasserstoff geschwängert war, und darauf getrocknet. Wieder in Salpetersäure aufgelöst und in der Wärme durch Aetzkali gefällt, gab er 0,120 Gr. Kupferoxyd.

Die Flüssigkeit, aus der das Kupferoxyd ansgeschieden war, wurde langsam abgedampft, mit einigen Tropfen Salpetersäure erhitzt und durch Ammoniak gefält. Das Eisenoxyd wurde lange gewaschen, nachber getrocknet und bei dem $\mathbf{Z n -}$ tritte der Luft bis zum starken Rothglühen erhitzt. Seine Menge belief sich auf $0,492 \mathrm{Gr}$.

Könnte man den Sauerstoff des Kupfer - und Eisenoxydes vereinigen, so würde man beinahe folgendes sehr einfache Verhältniss haben:

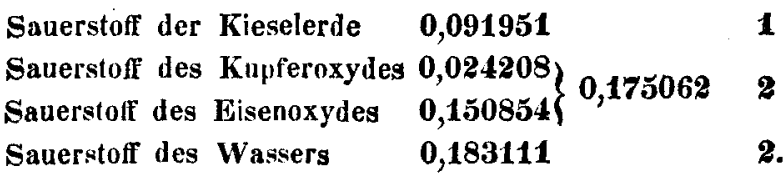

Da aber diese beiden Oxyde nicht die nämliche Formel haben, so laisst sich schwerlich eine Verbindung wie diese annehmen. Verbände man dagegen das ganze Eisenoxyd mit der Menge Wasser, die erforderlich ist, um die unter dem Namen Limonit bekannte Species auszumachen, so bätte man:

Journ. f, prakt. Chemie. XIII. 6. 
354 Damour, üb. cadmiumhalt. Schwefelzink.

\begin{tabular}{lc} 
Eisenoxyd & $\mathbf{0 , 4 9 2}$ \\
Wasser & $\mathbf{0 , 0 9 2}$ \\
\cline { 2 - 2 } Limonit & $\mathbf{0 , 5 8 4 .}$
\end{tabular}

Es würde nur noch übrig bleiben, das übrige Wasser mit der Kieselerde und dem Kupferoxyde zu verbinden, und man würde folgendes Verhältniss haben:

$\begin{array}{llll}\text { Kieselerde } & \mathbf{0 , 1 7 7} & \mathbf{0 , 0 9 1 9 5 1} & 4 \\ \text { Kupferoxyd } & \mathbf{0 , 1 2 0} & \mathbf{0 , 0 2 4 2 0 8} & 1 \\ \text { Wasser } & \mathbf{0 , 1 1 5} & \mathbf{0 , 1 0 2 2 2 1} & 4\end{array}$

Dieses Mineral würde dann die innige Verbindung des Limonites mit einem wasserhaltigen Kupferoxydsilicate von einer neuen Formel darbieten. Wie dem auch sei, so schien es mir eine besondere Species ausmachen zu müssen.

\section{XLVIII.}

Ueber das cadmizmhaltige Schwefelsink aus der Grube von Nuissière bei Bearjeu (Departement Rhône).

Von

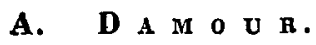

(Annales des Mines, troisième série, tome XII. 5te Lieferang. 1837. S. 245.)

Die Anwesenheit von Cadmium in gewissen Zinkmineralien aus Schlesien, Böhmen and Britannien ist schon verschiedene Male dargethan worden. Alles lässt glauben, dass dieses Metall an vielen andern Orten sich findet. Unter den verschiedenen mineralisshen Substanzen, die Danbauser aus der Bleigrube von Nuissière bei Beaujen mitbrachte, machte dieser Naturkundige mich auf eine Blende von röthlicher Farbe aufmerksam, die bald in Krystallen war, bald Adern in Quarz bildete und aus deren Aussehen er vermuthete, dass sie ein von dem Zink verschiedenes Metall enthalten könnte. Ein vor dem Löthrobre mit einigen Stücken des Minerals vorgenommener Versuch gestattete mir bald, darin die Anwesenheit von Cadmium zu erkennen. 